\title{
NEWS AROUND THE WORLD
}

\section{Generic Human Menopausal Gonadotropin (hMG) in Place of More Costly Follicle-Stimulating Hormone (FSH) for Routine Ovulation Induction ${ }^{1}$}

\author{
NORBERT GLEICHER ${ }^{2,3}$ and VISHVANATH KARANDE ${ }^{2}$
}

\begin{abstract}
Purpose: A large part of infertility treatment involves the use of exogenous gonadotropins. The last decade has seen a progressive switch from human menopausal gonadotropin ( $h M G)$, the original gonadotropin product, to progressively more costly products, primarily or exclusively containing follicle-stimulating hormone (FSH). Though obviously at least in part driven by marketing efforts of pharmaceutical companies, this switch has received relatively little scrutiny despite its obvious cost implications. We therefore investigated whether a switch back to a generic or less costly $h M G$ driven ovulation induction protocol would affect patient outcome after ovulation induction and, by implications, with other assisted reproductive technologies.
\end{abstract}

DISCLOSURE: Prior to submission of this manuscript, Dr. Gleicher has served as a consultant to Serono Laboratories, Randolph, MA, and has received consultation fees of $\$ 2,500.00$ as well as travel reimbursements for consultation meetings. He and Dr. Karande have performed investigational studies for Serono Laboratories as well as for Organon, Inc., West Orange, NJ, involving various of their gonadotropin products. None of the physicians received any personal payments for performance of the study. Payments were made to the Center for Human Reproduction (Illinois) of which Drs. Gleicher and Karande were shareholders at the time. Dr. Gleicher was sponsored for a travel grant of approximately $\$ 5,000.00$ by Ferring Pharmaceuticals, Tarrytown, NY, to speak at a scientific meeting on a topic unrelated to ovulation induction through gonadotropins. As Editor-in-Chief and Managing Editor of this journal, respectively, the opinions presented represent those of Drs. Gleicher and Karande only and not those of either the Editorial Board of this journal or its publisher. ${ }^{1}$ Supported in part by the Foundation for Reproductive Medicine, Chicago, Illinois.

${ }^{2}$ Center for Human Reproduction, Chicago, Illinois 60610.

${ }^{3}$ To whom correspondence should be addressed at Center for Human Reproduction,--Illinois, 750 North Orleans Street, Chicago, Illinois 60610.

\begin{abstract}
Methods: We prospectively studied clinical pregnancy rates in a large number of consecutive ovulation induction cycles in a well-defined patient population (group 1) which, after October of 1997, had been switched from a predominantly FSH to an hMG-driven protocol, based on an institutional formulary change. Until a transition period (between July and September 1997), this patient population had been on a primarily FSH-driven protocol (between July 1996 and June 1997). In parallel, we evaluated a second patient population (group 2), which was managed by the same physicians outside of formulary requirements and remained almost exclusively on principally FSH-driven ovulation induction cycles.
\end{abstract}

Results: FSH- and hMG-driven ovulation induction protocols did not differ in pregnancy outcome during the prospective study period. Group 1 patients, however, demonstrated a significant increase in pregnancy rates after the switch from FSH to hMG stimulation had taken place $(\mathrm{P}=0.02)$, while group 2 patients demonstrated no change in pregnancy rate during the same time period.

Conclusions: Generic hMG products do not adversely affect pregnancy rates in comparison to more costly FSH products in routine ovulation induction cycles and should be considered an appropriate alternative to more expensive FSH products.

KEY WORDS: Follicle stimulating hormone (FSH); ovulation induction; human menopausal gonadotropin.

\section{INTRODUCTION}

The cost of infertility treatment in the United States is considerable and by far exceeds similar treatment 
costs in other developed nations. One reason for this discrepancy lies in higher costs of pharmaceuticals, especially exogenous gonadotropins, which in the United States can reach many multiples of charges for the same products by the same manufacturers in other countries.

Exogenous gonadotropins represent a mainstay of infertility treatment in that they are essential for standard ovulation induction as well as in in vitro fertilization (1,2). Their cost structure, therefore, warrants long-overdue scrutiny and is the subject of this investigational study as well as the opinion piece by the two principal editors of this journal (please also note the Disclosure statement). Between 1994 and 1998 the cost of exogenous gonadotropins has dramatically increased, primarily driven by an active effort of the pharmaceutical industry to convert from human menopausal gonadotropin (hMG) to follicle-stimulating hormone (FSH) stimulation and later from biological, urinary derived to recombinant products (1).

The first clinically available preparations of exogenous gonadotropins were hMGs that consist of 75 IU FSH and 75 IU of luteinizing hormone (LH). A next generation of gonadotropins involved the so-called urofollitropins ( $\mathrm{uFSH}$ ), which contained a preponderance of FSH (75 IU) and only very little LH $(<0.7$ IU) per ampule. This was followed by highly purified FSH (pFSH), containing still 75 IU of FSH but less than 0.001 IU of LH per ampule, and finally and most recently by recombinant FSH (rFSH) which pharmacokinetically is most similar to $\mathrm{uFSH}$ (1).

The Center for Human Reproduction (CHR), Illinois' largest provider of infertility services during the study period, principally used hMG preparations for its ovulation induction program until July 1995. As a consequence of market shortages in the supply of hMG, a favorable pricing structure, availability of $\mathrm{uFSH}$, and finally a rather aggressive marketing drive by what then was the sole manufacturer of exogenous gonadotropins in the US market, CHR in August of 1995 replaced hMG with $\mathrm{uFSH}$ as its principal gonadotropin for ovulation induction. Subsequently, as further generations of FSH products entered the market, they were progressively integrated to replace earlier FSH products.

Following the replacement of hMG by $\mathrm{uFSH}$, a decline in the CHR's pregnancy rates following ovulation induction with gonadotropins was suspected based on monthly generated outcome statistics. Since the published literature contradicted such an associa- tion $(1,3)$, the change in pregnancy rates was attributed to the merger of a number of practices into CHR during 1995 and a possible consequential change in patient population (4). Overall pregnancy rates after ovulation induction with gonadotropins remained at a stable level through mid-1997, when the introduction of a significantly less costly generic $\mathrm{hMG}$ product to the US market raised the question whether CHR should reintroduce the usage of hMG into its ovulation induction program.

A careful review of the literature led to the conclusion [recently confirmed in a Practice Committee Report by the American Society of Reproductive Medicine (1)] that there appears to be no advantage of one preparation of exogenous gonadotropin over another for ovulation induction (with the exception of woman with hypogonadotropic hypogonadism). Consequently, CHR established this new and significantly less costly hMG product as its primary gonadotropin for ovulation induction on the formulary as of late summer of 1997.

This meant that all patients for which CHR provided gonadotropins under contractual obligations (4) received hMG exclusively, unless an FSH product was clinically indicated. Patients who received care at CHR outside of such contractual agreements, and thus were not part of a utilization review process, could receive either hMG or FSH products, depending on their physicians' preference.

Because the Formulary Committee of CHR was concerned that, despite no data to the contrary, the switch to hMG may adversely affect the pregnancy chances of the contracted patient population, the Department of Statistics at CHR (as part of the center's continuous quality improvement program) was charged with reporting to the physicians a prospective month-to-month follow-up of outcome, comparing the hMG-treated contracted patient populations with a control population under other treatment alternatives. The purpose of this somewhat more detailed follow-up on outcome than was usually conducted was to recognize any adverse outcome changes that may arise as a consequence of the switch to the less costly hMG product. Any such negative effect would immediately have resulted in a return to routine FSH stimulation for the contracted patient population. The here-presented study reports on this follow-up.

\section{MATERIALS AND METHODS}

CHR was at the time of the study, Illinois' largest 
provider of infertility services seeing approximately 3500 new patient couples per year. Among those, approximately one third were insured by the state's largest health maintenance organization (HMO), a Blue Cross/Blue Shield of Illinois affiliate, called HMO-Illinois (HMO-I). CHR held an exclusive provider contract for comprehensive infertility services since 1993 (4). Under this contract, HMO-I patients had to receive female and male infertility services through CHR if they were to be covered by the insurance carrier. HMO-I membership increased from 350,000 in 1994 to 641,921 in April 1998. During the same time period patients received a surprisingly persistent level of services, based on services billed at full fee per member per month (PMPM) or per couple per month (PCPM) (Table I). Concomitantly, selective enrollment into HMO-I resulted in an increase of patients seeking treatment from 1.39/1,000 to 1.73/1,000 between 1994 and 1997, and medication usage, based on full fee, increased from $\$ 0.41$ to $\$ 0.51$ PMPM but remained stable at $\$ 341.17$ to $\$ 337.43$ PCPM (Table I). During the same time period, the distribution of underlying causes for infertility did not change significantly (data not shown).

Between 1994 and June 1997, hMG was progressively replaced by FSH as the principal gonadotropin. After mid-1997, a reverse switch took place. The cycle statistics represent consecutive ovulation induction cycles under gonadotropin stimulation performed during these time periods. In excess of $90 \%$ of cycles were accompanied by intrauterine inseminations (IUIs) and practice patterns remained consistent at CHR during these time periods.

Ovulation inductions were conducted in routine fashion (1), whether under hMG or FSH stimulation. IUIs were performed as previously reported (5).
Patients who qualified for ovulation induction treatment fell into World Health Organization (WHO) classes 1 and 2 (1) and usually had failed prior ovulation induction efforts with clomiphene citrate for at least three cycles. Patients received ovulation induction with gonadotropin only for a maximum of four cycles. This treatment algorithm was contractually determined with HMO-I but was uniformly applied to all patients within the CHR system (4). Patients who did not conceive with up to four gonadotropin cycles were advanced into in vitro fertilization, which in Illinois has been a covered insurance benefit under a state mandate (4).

Every treatment cycle at CHR is entered into a fully computerized data bank accessible to all staff. Because this data bank also serves as a paperless record for purposes of cycle monitoring, all data are entered in real time (6). The center's continuous quality improvement program also uses these data to generate a monthly outcome report on basically all treatments. Physicians practice individually, though agreement existed on standard treatment protocols. Moreover, daily cycle monitoring is often conducted online and in groups, thus establishing a reasonable uniformity of care within CHR. The data are statistically summarized in monthly intervals by a medical statistician in the Department of Medical Statistics and reported out to all physicians who thus have feedback on outcomes (6).

An initial switch from an hMG product (Pergonal, Serono Laboratories, Randolph, MA) to a uFSH product (Metrodin, Serono Laboratories, Randolph, MA) took place in the summer of 1995. By September of 1995, in excess of $75 \%$ of gonadotropin cycles were conducted using predominantly or exclusively a uFSH product. During 1995, the same manufac-

Table I. Characteristics of Study Population

\begin{tabular}{|c|c|c|c|c|c|}
\hline & \multicolumn{5}{|c|}{ YTD } \\
\hline & 1994 & 1995 & 1996 & 1997 & 1998 \\
\hline $\begin{array}{l}\text { Membership on January } 1 \\
\text { (in thousands) }\end{array}$ & 350 & 376 & 406 & 491 & 610 \\
\hline $\begin{array}{l}\text { New patients per } 1000 \\
\text { service charges }\end{array}$ & 1.39 & 1.61 & 1.51 & 1.73 & N.A..$^{a}$ \\
\hline $\mathrm{SPMPM}^{b}$ & 2.13 & 2.08 & 1.88 & 1.89 & 1.90 \\
\hline $\mathrm{SPCPM}^{c}$ & 1769 & 1468 & 1278 & 1254 & 1312 \\
\hline Medication charges & & & & & \\
\hline SPMPM & 0.41 & 0.56 & 0.48 & 0.51 & 0.51 \\
\hline SPCPM & 341.17 & 393.79 & 326.65 & 337.43 & 355.28 \\
\hline
\end{tabular}


turer introduced an FSH product of improved purity (Fertinex, Serono Laboratories, Randolph, MA) and later replaced Metrodin completely in the US market with this more costly and more pure product (pFSH) in early 1997. CHR switched its FSH products in parallel. In late 1997, the same pharmaceutical company entered the market with a recombinant product (Gonal-F, Serono Laboratories, Randolph, MA) and shortly thereafter a competing recombinant product became available (Follistim, Organon, Inc., West Orange, NJ). CHR only selectively integrated the use of both these recombinant products into its practice, since both products were significantly more expensive than the prior generation of $\mathrm{FSH}$ products.

By the summer of 1997, a third gonadotropin manufacturer received FDA approval for an hMG gonadotropin product (Repronex, Ferring Pharmaceuticals, Tarrytown, NY). This product was significantly less costly than any of the competing FSH products and was therefore established as the primary gonadotropin product on the CHR formulary in the summer of 1997. By October 1998, 88\% of all ovulation induction cycles in HMO-I patients were conducted under hMG stimulation. For the last quarter of 1997 (October-December) only 8\% of cycles were conducted under predominant or exclusive FSH stimulation. All these cycles had specific clinical indications for FSH usage, such as polycystic ovarian disease, ovarian resistance to stimulation, or high day-3 FSH levels (1-3). This pattern of predominant hMG utilization has continued for HMO-I patients through April 1998.

HMO-I patients receive their injectable pharmacy benefits through CHR. Basically all other CHR patients do not. Because CHR's contract with HMO-I was a capitated risk contract, all medication usage undergoes utilization review (4). Other patients' cycles, however, did not. Moreover, physicians had free choice of medication use in those other cycles.

In contrast to HMO-I cycle treatments, the availability of a relatively inexpensive gonadotropin product did not affect historical usage patterns of gonadotropins in non-HMO-I patients. While this came as a surprise to the medical leadership of CHR, it created a prospective control group of non-HMO-I patients who were treated by the same 14 physicians, under identical treatment protocols and algorithms, with identical cycle monitoring processes conducted by the same nursing staff, and under identical statistical outcome assessments. The only variation between
HMO-I (group 1) and non-HMO-I (group 2) patients was in the principal gonadotropin stimulation with hMG (group 1) and FSH (group 2), respectively, with the latter representing over $90 \%$ of cycles under FSH stimulation.

As noted previously, patients reached gonadotropin stimulation only after they exhausted at least three cycle stimulations with clomiphene citrate or demonstrated other clinical contraindications to clomiphene citrate, such as advanced female age or high day-3 FSH levels.

Statistical analysis was performed using chi-square analysis and $95 \%$ confidence intervals. Statistical significance was assumed at $P<0.05$.

Because this study involved only the summation of routine outcome data generated by a monthly continuous quality improvement effort and did not involve any experimentation, it was not judged to require review and approval by the institutional review board (IRB). The CHR's IRB does, however, at regular intervals review selected and relevant clinical outcome data generated by the monthly continuous quality improvement process, primarily as the data relate to the center's assisted reproductive technologies (ART) program, which is conducted under IRB supervision.

\section{RESULTS}

Outcome data were comparatively analyzed in three time periods. The historical period (phase A) was defined as the treatment period between January 1, 1994 and June 30, 1997. It involved 1833 consecutive cycles in group 1 and 3048 consecutive cycles in group 2. Phase $\mathrm{A}$ was subdivided into phase $\mathrm{A}_{1}$ (January 1, 1994 to June 30, 1995), the historical hMG treatment period, phase $\mathrm{A}_{2}$ (July 1, 1995 to June 30, 1996), a historical transition period from hMG to FSH stimulation, and phase $\mathrm{A}_{3}$ (July 1, 1996 to June 30, 1997), a predominant FSH treatment period. Phase B represented the transition period from FSH predominance to hMG predominance between July 1, 1997 and September 30, 1997 involving 169 group 1 and 266 Group 2 cycles, whereas phase C referred to the prospectively dominant usage of hMG between October 1, 1997 and April 30, 1998 in 369 group 1 and 564 group 2 cycles.

Group 1 pregnancy rates did not vary between phases A and B. They demonstrated, however, a significant improvement in phase $\mathrm{C}$ over phase $\mathrm{A}_{3}$ $(P=0.02)$. In contrast, group 2 patients did not show 
variation in pregnancy rates between phases $\mathrm{A}, \mathrm{B}$, or C (Table II).

Pregnancy rates in group 1 patients did not differ significantly from those of group 2 patients in any study period (Table II).

\section{DISCUSSION}

This study evolved out of the classic need in modern health care to control cost. Although the need to control cost exists throughout medicine, it is especially urgent in areas of medicine where excessive cost may prevent patients from receiving care at all. Infertility is such an area of medicine, since a large majority of couples with infertility do not receive insurance coverage through their health plans (7), and therefore have to fund infertility care out of pocket. Where insurance coverage is offered, often as a consequence of legislative mandates, preferred providers may be forced to assume cost risks, as was the case in the study here reported $(4,7)$.

Because medication costs can represent 30 to $50 \%$ of total treatment costs (N. Gleicher, unpublished data), cost-effective utilization of pharmaceuticals in infertility care is of utmost potential importance.

$\mathrm{CHR}$, which during the here-reported study period carried a capitated risk contract for in excess of
600,000 persons, on an ongoing basis carefully evaluated medication use through a utilization control system. When, through the entry of a new manufacturer into the US market, a significantly less costly generic exogenous gonadotropin became available, after careful consideration, the Formulary Committee of CHR reached the conclusion that use of this significantly less costly generic hMG product in women who had no specific indication for FSH usage should not adversely affect pregnancy rates during ovulation induction cycles. The committee nevertheless insisted on prospectively monitoring the capitated patient population month by month to confirm that a switch from primarily $\mathrm{FSH}$ - to hMG-driven stimulation would not result in any unexpected adverse outcomes.

Such adverse outcomes could include not only lower pregnancy rates, but also an increase in patient complaints due to intramuscular (for hMG) rather than subcutaneous (for most FSH preparation) administration and an increase in allergic reactions with hMG over FSH, since FSH products are either more purified or recombinant in nature (1).

This prospective evaluation of hMG treatment demonstrated excellent pregnancy rates and excellent clinical tolerance of the hMG product.

As "an experiment of nature," most CHR physicians failed to convert noncapitated (group 2) pa-

Table II. Statistical Comparison of Pregnancy Rates Between Groups 1 and 2 at Various Study Periods

\begin{tabular}{|c|c|c|c|c|c|}
\hline & Pregnant & Not pregnant & Odds ratio & $95 \% \mathrm{CI}$ & Chi-square \\
\hline \multicolumn{6}{|l|}{$A_{1}$} \\
\hline I & 109 & 689 & 1.04 & $0.78-1.4$ & NS \\
\hline II & 115 & 758 & & & \\
\hline \multicolumn{6}{|l|}{$\mathrm{A}_{2}$} \\
\hline I & 63 & 436 & 0.78 & $0.57-1.08$ & NS \\
\hline II & 173 & 938 & & & \\
\hline \multicolumn{6}{|l|}{$\mathrm{A}_{3}$} \\
\hline $\mathrm{I}$ & 56 & 480 & 0.8 & $0.56-1.12$ & NS \\
\hline II & 136 & 928 & & & \\
\hline \multicolumn{6}{|l|}{$\mathrm{B}^{11}$} \\
\hline I & 21 & 148 & 1.04 & $0.55-1.94$ & NS \\
\hline II & 32 & 234 & & & \\
\hline \multicolumn{6}{|l|}{$\mathrm{C}^{11}$} \\
\hline I & 59 & 310 & 1.22 & $0.83-1.8$ & NS \\
\hline II & 76 & 488 & & & \\
\hline Group 1 & & A vs. C & 0.75 & $0.54-1.83$ & NS \\
\hline Group 1 & & $A_{1}$ vs. $A_{2}$ & 1.36 & $0.95-1.94$ & NS \\
\hline Group 1 & & $\mathrm{~A}_{3}$ vs. $\mathrm{C}$ & 0.6 & $0.41-.93$ & 0.02 \\
\hline Group 1 & & $\mathrm{~A}_{1}$ vs. $\mathrm{C}$ & 1.11 & $0.66-1.9$ & NS \\
\hline Group 1 & \multicolumn{3}{|c|}{ A vs. $\mathrm{B}$ vs. $\mathrm{C}$} & \multicolumn{2}{|c|}{$P=0.175(\mathrm{NS})$} \\
\hline Group 2 & \multicolumn{3}{|c|}{ A vs. B vs. C } & \multicolumn{2}{|c|}{$P=0.682(\mathrm{NS})$} \\
\hline Group 1 & \multicolumn{3}{|c|}{$A_{1}$ vs. $A_{2}$ vs. $A_{3}$} & \multicolumn{2}{|c|}{$P=217(\mathrm{NS})$} \\
\hline Group 1 & \multicolumn{3}{|c|}{$\mathrm{A}_{1}$ vs. $\mathrm{A}_{3}$ vs. $\mathrm{C}$} & \multicolumn{2}{|c|}{$P<0.001$} \\
\hline
\end{tabular}


tients to the new hMG product and persisted in this patient population with an almost exclusively FSHdriven stimulation protocol. The more expensive pharmaceutical treatment approach did not, however, prove to be superior. In fact, pregnancy rates in group 2 stagnated, while group 1 patients appeared to demonstrate a significant improvement as a consequence of the switch in gonadotropin use that took effect between phase $A_{3}$ and phase $C$.

The purpose of this study was not to either support or prove superiority of hMG over FSH stimulation during routine ovulation induction. Indeed, the careful follow-up was performed to reassure the Formulary Committee about replacing routine FSH with routine hMG stimulation. The committee had come to such an approach based on a careful review of the published literature. Since then, the American Society of Reproductive Medicine in a Practice Committee Report confirmed our committee's conclusion that in fact in a large majority of women there appears to be no advantage of one preparation of exogenous gonadotropins over another for ovulation induction (1).

The finding of a statistically significant improvement in pregnancy rates in group 1 patients with hMG stimulation therefore came as a surprise, should be viewed with caution and calls for a possible explanation.

Because this study was not prospectively randomized, one such possible explanation is that a program as large and multifaceted as CHRs cannot rule out the existence of unrecognized confounding factors that contributed to an improved pregnancy rate in group 1. Such an explanation is unlikely, however, since any change in program activity should have similar effects on groups 1 and 2. After all, groups 1 and 2 were treated at the same time for the same conditions by the same physicians and staff in the same office locations of CHR under identical treatment protocols. The only detectable difference was the primary of FSH (group 2) versus hMG (group 1) stimulation.

In accepting the principal limitation of this study (that it was not prospectively randomized), one is left with the question whether there might be a physiological explanation why the induction of ovulation with hMG may improve pregnancy rates over a standard induction protocol with $\mathrm{FSH}$.

Such an explanation, in fact, exists. Based on Armstrong's (8) two cell, two gonadotropin theory, LH is necessary to stimulate androgen production by theca cells, whereas FSH stimulates granulose cells to con- vert androgens to estrogen, if normal ovulation is to take place. More recently, however, it has been suggested that FSH alone can stimulate normal follicular function if circulating adrenal, constitutive, or FSH-stimulated thecal androgens are available to produce intrafollicular estradiol (9). Such exclusively FSH-driven folliculogenesis, however, may not be possible in all females. Because newer-generation FSH products are either highly purified urinary products or produced through recombinant techniques, their LH content is either zero or close to zero (1). Such FSH products therefore may be inadequate for a small minority of women who do require small amounts of exogenous LH during ovulation induction. A study involving large cycle numbers, as presented here, may detect the results of inadequate follicular maturation as a consequence of $\mathrm{LH}$ deprivation even in only a relatively small minority of women. In fact, if only approximately $10-20 \%$ of women were to be affected by poor follicular maturation, the improvement in pregnancy rates observed in this study in group 1 already could be explained.

Sauer et al. recently observed that ovarian stimulation with rFSH after down-regulation with a gonadotropin-releasing hormone ( $\mathrm{GnRH})$ agonist resulted in follicular arrest in up to $20 \%$ of rhesus monkeys (M. Sauer, New York, NY, personal communication). The same investigators also experienced a cycle cancellation rate in the range of 10 to $20 \%$ in young, healthy oocyte donor when stimulated with rFSH after down-regulation with a GnRH-agonist. Similar donor populations had experienced less than 5\% cycle cancellation when stimulated with less pure exogenous FSH products (M. Sauer, New York, NY, personal communication). These observations strongly suggest that at least in some patients the routine use of pure or relatively pure FSH products may not be advisable without the addition of at least small dosages of LH.

Whether routine hMG stimulation is superior to routine FSH stimulation, however, is not the principal issue addressed in this study. This study mostly suggests that routine ovulation induction can be performed cost-effectively with generic hMG products. This is best demonstrated by the fact that at no point during the almost 4-year study period did group 1 patients differ in pregnancy rates from group 2 patients. This lack of statistical difference was maintained whether both groups of patients were treated with identical gonadotropin products or diverged as in phase $\mathrm{C}$. Considering the price advantage of generic hMG over currently existing FSH products on 
the US market, such a treatment approach may save in excess of one third of medication costs.

It is unknown how many gonadotropin-induced ovulation induction cycles are performed annually in the United States. Considering a conservative number of 100,000 such cycles [59, 142 in vitro fertilization and other ART cycles were performed in the United States during 1995 (10)] and assuming an average usage of $225 \mathrm{IU}$ (three ampules) for 9 days of stimulation, over $\$ 40$ million in pharmaceutical cost could be saved. At a capitation rate of $\$ 1.25$ PMPM for comprehensive infertility coverage ( $\$ 15.00$ per year), these savings could pay for an additional 2.7 million people's infertility coverage for 1 year or could cover full infertility services on a capitation or case-rate basis for approximately 4670 additional couples at a utilization of 1.73/1000 insured persons (the utilization rate encountered during 1997 in this study).

\section{REFERENCES}

1. American Society for Reproductive Medicine Practice committee report: Induction of ovarian follicle development and ovulation with exogenous gonadotropins, 1998, pp 1-11

2. Jones HW, Toner JP: The infertile couple. New Engl J Med 1993;329:1710-1715
3. Venturoli S, Paradisi R, Fabbri R, et al.: Comparison between human urinary follicle-stimulating hormone and human menopausal gonadotropin treatment in polycystic ovary. Obstet Gynecol 1984; 63:6-11

4. Pratt DE, VanderLaan BF, Dudkiewicz A, et al.: A managed care provider's approach towards mandated infertility coverage: the Illinois Family Building Act. J Assist Reprod Genet 1994; 11:433-438

5. Karande VC, Rao R, Pratt D, et al.: A randomized prospective comparison between intrauterine insemination and fallopian sperm perfusion for the treatment of infertility. Fertil Steril 1995; 654:638-640

6. Pratt DE: A computer system for outcome assessment and utilization review in reproductive medicine. Infertil Reprod Med Clin North Am 1998; 9:85-96

7. Cohen AW: Managed healthcare's approach to infertility. Infertil Reprod Med Clinics North Am 1998;9:21-30

8. Armstrong DT, Coff AK, Dorrington JH: Regulation of follicular estrogen biosynthesis. In Ovarian Follicular Development and Function, AR Midgley, WA Sadler, (eds.), New York, Raven Press, 1979, pp 159-182

9. Ben-Chetrit A, Gotlieb L, Wong PY, et al.: Ovarian response to recombinant human follicle-stimulating hormone in luteinizing hormone-depleted women: examination of the two cell, two gonadotropin theory. Fertil Steril 1998;69:59S-65S

10. Society for Assisted Reproductive Technology and the American Society for Reproductive Medicine: Assisted reproductive technology in the United States and Canada: 1995 results generated from the American Society for Reproductive Medicine/ Society for Assisted Reproductive Technology Registry. Fertil Steril 1998;69:389-398 